\title{
Métodos contraceptivos reversíveis de longa duração no Sistema Único de Saúde: o debate sobre a (in)disciplina da mulher
}

\author{
Long-acting reversible contraception methods in the Brazilian \\ Unified National Health System: the debate on women's \\ (in)discipline
}

${ }^{1}$ Instituto de Estudos em Saúde Coletiva, Universidade Federal do Rio de Janeiro. Praça Jorge Machado Moreira 100, Cidade Universitária. 21941 590 Rio de Janeiro RJ Brasil.brandao@iesc.ufrj.br

\begin{abstract}
Recently, the Brazilian Federation of Gynecology and Obstetrics Associations submitted a request to the Brazilian Ministry of Health for an introduction of long-acting reversible contraception (LARC) methods for young women aged 15 to 19 years in the Brazilian Unified $\mathrm{Na}$ tional Health System. The two devices to be included were the etonogestrel-releasing subdermal implant (ENG implant), with a duration of three years, and the levonorgestrel-releasing intrauterine system (LNG-IUS), lasting five years. The Ministry of Health then launched a public inquiry to evaluate this introduction, deciding against the inclusion of these methods in the public health services. The article discusses the discursive strategies used to justify the acceptance and applicability of these methods in "special populations." The debate on family planning needs to understand fully the discontinuity of contraception in the use of such methods, the central concept of emergency contraception, and how gender hierarchies prejudice safe contraceptive practice. On the contrary, the emphasis on the (in)discipline of women regarding care with regular-use contraceptive methods effectively reinforces their condition of social minority.
\end{abstract}

Key words Sexuality, Gender, Contraceptive methods, Family planning, Long-acting reversible contraception (LARC) methods
Resumo Recentemente a Federação Brasileira das Associações de Ginecologia e Obstetrícia submeteu ao Ministério da Saúde uma solicitação para oferta no Sistema Único de Saúde de métodos contraceptivos reversiveis de longa duração (LARC), para jovens mulheres de 15 a 19 anos. $O s$ dois dispositivos a serem incluídos seriam o implante subdérmico liberador de etonogestrel, com duração de três anos, e o sistema intrauterino liberador de levonorgestrel, com duração de cinco anos. O Ministério da Saúde abriu então consulta pública para avaliar tal introdução, terminando por decidir contrariamente à inclusão destes métodos na rede pública de saúde. O artigo discute as estratégias discursivas utilizadas para fundamentar e justificar a aceitação e aplicabilidade destes métodos em "populações especiais". O debate sobre o planejamento reprodutivo precisa compreender melhor as descontinuidades contraceptivas no uso de métodos, a centralidade da contracepção de emergência e o quanto as hierarquias de gênero dificultam uma prática contraceptiva segura. Ao contrário, a ênfase na (in) disciplina da mulher no tocante aos cuidados com a utilização de métodos contraceptivos de uso regular termina por reforçar sua condição de menoridade social.

Palavras-chave Sexualidade, Gênero, Métodos anticoncepcionais, Planejamento familiar, Métodos contraceptivos reversiveis de longa duração (LARC) 


\section{Introdução}

Usualmente, a questão do planejamento reprodutivo e da ocorrência de gravidez imprevista no Brasil tem sido um tema delicado e controverso ${ }^{1}$. Em um país onde o aborto continua sendo interditado às mulheres, embora seja uma prática bastante frequente e realizada em condições inseguras $^{2}$, sofremos ainda com o surgimento de epidemias como a do zika vírus, que além de espelhar magistralmente as desigualdades sociais, afeta de modo dramático particularmente as mulheres jovens, grávidas e de regiões empobrecidas no norte e nordeste do país ${ }^{3,4}$.

Analisando os dados da última Pesquisa Nacional de Demografia e Saúde da Criança e da Mulher (PNDS), realizada em 2006, Perpétuo e Wong $^{5}$ ressaltam uma ampliação do uso de anticoncepcionais no país em relação à pesquisa anterior de 1996, com redução significativa do recurso à laqueadura tubária, entre mulheres de 15 a 44 anos, em união conjugal. No entanto, a esterilização feminina $(25,9 \%)$ e a pílula oral $(27,4 \%)$ continuam sendo os dois métodos mais prevalentes na população. $\mathrm{O}$ uso de preservativo masculino cresceu $(13,0 \%)$ e a contracepção de emergência aparece como quinto método mais usado entre todas as mulheres em idade reprodutiva. Decerto que dez anos depois, provavelmente esse cenário já se transformou e pode apontar alterações significativas nas práticas contraceptivas femininas na última década.

A discussão sobre qual método contraceptivo (hormonal ou não) é apropriado a cada mulher, em cada fase de seu ciclo de vida e em condições específicas de parceria sexual (ocasional, estável, múltiplas) é circunstancial e precisa ser ponderada em cada contexto cultural particular. Assim, as ações de aconselhamento, orientação, avaliação clínica e acompanhamento de saúde são imprescindíveis para que cada usuária do Sistema Único de Saúde (SUS) se sinta amparada em suas escolhas e obtenha acesso aos métodos no tempo oportuno.

\section{Inclusão de métodos contraceptivos reversíveis de longa duração no Sistema Único de Saúde}

A introdução de uma ampla e variada oferta de alternativas contraceptivas na rede pública de serviços de saúde é sempre uma premissa importante que aumenta as possibilidades de atendimento de necessidades diferentes. Do contrário, as usuárias são levadas à compra de contracep- tivos em drogarias, nem sempre acompanhada de orientação farmacêutica adequada. Na ultima PNDS $2006^{6}$, as farmácias aparecem como fonte de obtenção de métodos contraceptivos modernos para 42,5\% das usuárias de 15 a 49 anos, reunindo os métodos hormonais (pílula e injeção) e preservativo masculino.

Assim, as recentes consultas públicas disponibilizadas pela Comissão Nacional de Incorporação de Tecnologias no SUS (CONITEC), n. ${ }^{\circ} 35$ e n. ${ }^{\circ} 36^{7}$, em 2015 , sobre uma eventual introdução no Sistema Único de Saúde (SUS) dos métodos contraceptivos reversíveis de longa duração (Long-acting reversible contraception, LARC), solicitadas pela Federação Brasileira das Associações de Ginecologia e Obstetricia (FEBRASGO) ao Ministério da Saúde (MS), atualizam o debate a respeito da inclusão de novas alternativas contraceptivas às mulheres.

Trata-se da oferta de dois dispositivos contraceptivos às mulheres entre $15 \mathrm{e} 19$ anos: o implante subdérmico liberador de etonogestrel, com duração de três anos, e o sistema intrauterino liberador de levonorgestrel, com duração de cinco anos. A Federação defende a introdução dessa modalidade de contraceptivos na rede pública de saúde, com indicações para uso em "populações especiais", como adolescentes, usuárias de drogas ilícitas e mulheres convivendo com vírus da imunodeficiência humana ${ }^{8}$. Em outro artigo ${ }^{9}$, os grupos de mulheres em situação de vulnerabilidade são detalhados: "estão incluídas as de baixa renda e escolaridade, as moradoras de áreas rurais ou regiões isoladas e remotas, as privadas de liberdade, as que vivem com vírus da imunodeficiência humana (HIV) ou com outras doenças crônicas, como epilepsia, bem como as indígenas, as adolescentes, as usuárias de drogas ilícitas e as portadoras de deficiência física e/ou mental".

Repercutindo um debate internacional sobre os métodos reversíveis de longa duração (LARC) $)^{10,11}$, a defesa da introdução desta modalidade de método contraceptivo para o amplo acesso das jovens usuárias brasileiras, destaca as elevadas taxas de gravidez imprevista no país ${ }^{12}$, a vulnerabilidade de determinados estratos sociais e a alegação de que tais métodos "não dependem da disciplina da mulher"13. Apoiando-se na possibilidade de falhas, esquecimentos, interrupções no uso contínuo dos métodos hormonais disponíveis (púlulas orais combinadas, injetáveis mensais ou trimestrais), os argumentos em prol dos LARC destacam: "Uma das principais vantagens dos LARCs em relação aos contraceptivos reversíveis de curta duração é a manutenção da sua 
alta eficácia, independentemente da motivação da usuária. Devido à facilidade de uso, os LARCs ganharam o apelido 'pegue-o e esqueça-o"'8. Esse será o aspecto central a ser analisado, mas antes, é preciso esclarecer sobre o posicionamento do Ministério da Saúde diante de tal consulta.

Como resposta, em 2016, o Ministério da Saúde se coloca contrário à solicitação feita pela Febrasgo, pois "consideraram que as evidências científicas apresentadas não foram suficientes para comprovar superioridade da tecnologia proposta, comparada às tecnologias disponibilizadas no SUS. Além disso, a incorporação dos LARCs geraria um impacto orçamentário considerável em cinco anos, sem que algum benefício clínico tenha sido demonstrado" 14 .

\section{Sobre a (in)disciplina da mulher}

Independente da resposta negativa do $\mathrm{Mi}$ nistério da Saúde ao pleito, a discussão sobre as indicações clínicas e vantagens dos LARC cresce a cada dia no país ${ }^{15}$. Tais métodos já se encontram disponíveis na prática médica privada de ginecologistas nos grandes centros urbanos. Não creio que tal posicionamento deva encerrar o necessário debate público a respeito da utilização dos LARC no Brasil, em especial entre o público adolescente. Muito ao contrário, é preciso fomentar essa discussão para que atores institucionais envolvidos possam conhecer melhor o tema e participar do debate, sempre na perspectiva de defesa dos direitos sexuais e reprodutivos de adolescentes e jovens. A desigualdade social no acesso a métodos contraceptivos reversíveis de alto custo, assim como ocorre no acesso das mulheres às práticas seguras de abortamento, continuam penalizando mulheres pobres e negras, o que precisa ser corrigido. Neste sentido, a demanda da FEBRASGO ao MS e a consulta pública dela decorrente sinalizam a necessidade de uma discussão que inclua diversos olhares sobre a questão do planejamento reprodutivo no Brasil.

No entanto, o pleito nos obriga a refletir criticamente sobre as estratégias de apresentação destes novos produtos à sociedade, com vista a sua aceitação e aplicabilidade no Sistema Único de Saúde. Há um processo discursivo que constrói a mulher jovem como socialmente incapaz de se cuidar ou de seguir sem interrupções as prescrições médicas relativas ao uso de métodos contraceptivos. A justificativa que prioriza as "populações especiais" citadas (os conhecidos "grupos de riscos") reforça uma certa alienação social que seria inerente a essas mulheres, em es- pecial as adolescentes em situação de vulnerabilidade social.

As ideias veiculadas de que os LARC não dependem da usuária, "não dependem da disciplina da mulher", podem ser "esquecidos" após inseridos ofusca o necessário autocuidado com a contracepção que toda mulher precisa ter. A ideia de delegar ao médico a tarefa de escolha de um método contraceptivo (reversível ou não) minimiza a capacidade de escolha da mulher e seu poder de decisão como uma pessoa autônoma, capaz de avaliar e decidir naquele momento de vida a alternativa contraceptiva que melhor lhe atenda. Tampouco os LARC prescindem de acompanhamento clínico durante os anos em uso, para avaliar efeitos colaterais ou outro problema que possa surgir, em se tratando de métodos hormonais.

Decerto os altos percentuais de falhas contraceptivas, de esquecimento ou não uso de métodos contraceptivos em todas as relações sexuais compromete a eficácia dos métodos em uso, trazendo a possibilidade de uma gravidez ocorrer, sem ser esperada. Estudando o tema do "fracasso" contraceptivo, ou o que designa como descontinuidades contraceptivas, Moreau et al. ${ }^{16,17}$ assinalam que tais interrupções no uso consistente de contraceptivos podem estar relacionadas com as insatisfações das mulheres com os métodos em uso, entre outros aspectos. No caso das adolescentes, também podem estar relacionadas com a instabilidade das parcerias sexuais e/ou amorosas, à falta de apoio parental, à ausência desta discussão entre pares no ambiente escolar, à recusa dos parceiros em usar o preservativo, dentre outras razões. É preciso compreender que a adesão ao método contraceptivo não se coloca como uma decisão meramente individual, mas forjada sobre determinadas circunstâncias sociais que amparam ou não tais escolhas.

Decorre daí a importância de existir há aproximadamente vinte anos no Brasil a alternativa da contracepção de emergência (levonorgestrel), extensiva não somente às vítimas de violência sexual mas a todas as mulheres que tiveram uma relação sexual sem proteção ou interromperam por algum motivo o uso do método de rotina. Embora a contracepção de emergência não seja um método consensual no país e desperte muitas dúvidas e controvérsias por ser um método pós-coito ${ }^{18,19}$, se utilizada no tempo devido, logo após a ultima relação sexual sem proteção (até 120h) sua eficácia aumenta. Evidências científicas disponíveis demonstram que ela é um método seguro e com eficácia comprovada, se usada devidamente $\mathrm{e}^{20,21}$. 
No entanto, as dificuldades de acesso à contracepção de emergência na rede pública de serviços de saúde em tempo oportuno são imensas ${ }^{22}$, obrigando as usuárias a obtê-la nas drogarias, sem amparo devido e orientação de profissionais de saúde.

O crescimento da utilização da contracepção de emergência no Brasil, na última década, ainda é desconhecido, haja vista a ultima PNDS ter sido realizada em 2006 e já apontar crescimento do uso deste método no país entre mulheres jovens. No Brasil, 12\% das mulheres de 15 a 49 anos, sexualmente ativas, que já haviam utilizado um método contraceptivo moderno algum vez na vida, afirmaram ter usado a contracepção de emergência, segundo a PNDS de $2006^{6}$. Este percentual de uso da contracepção de emergência sobe para $23,2 \%$ se considerarmos apenas as mulheres não unidas. A PNDS 2006 aponta que a contracepção de emergência passou a ocupar o quinto lugar entre os métodos contraceptivos modernos usados por todas as mulheres e é o terceiro dentre os métodos modernos usados por mulheres não unidas e ativas sexualmente (últimos 12 meses). O uso é maior entre os grupos de idades mais jovens, correspondendo a $10,4 \%$ entre adolescentes de 15 a 19 anos que já utilizaram algum método moderno alguma vez na vida; $18,5 \%$ entre jovens de 20 a 24 anos e $16,9 \%$ entre jovens de 25 a 29 anos.

Estima-se que tal uso tenha aumentado significativamente em razão das mudanças socioculturais nas formas de interação sexual entre homens e mulheres - hoje mediadas pela tecnologia digital e mais imprevisíveis - e na constituição de parcerias mais ocasionais, muitas vezes, sem vínculo afetivo-sexual entre o casal. Pesquisa sobre práticas contraceptivas realizada em 2015, com mulheres de 15 a 44 anos no município de São Paulo ${ }^{23}$, mostrou que o crescimento da utilização da contracepção de emergência foi bastante expressivo em contraste aos outros métodos disponíveis. A prevalência de anticoncepção é bastante elevada $(84,3 \%)$ no município. O recurso à contracepção de emergência tem sido bastante frequente, particularmente entre mulheres com menos de 35 anos. Mais de $60 \%$ das mulheres com 15 a 29 anos de idade disseram já ter usado a contracepção de emergência pelo menos uma vez na vida.

A discussão sobre aceitação ou pertinência dos métodos de contracepção não pode ser desvinculada das práticas sexuais e das relações de gênero que mediam o contexto social dos sujeitos (homens e mulheres). As desigualdades e a violência de gênero são os maiores impedimentos para uma prática contraceptiva regular, sem atropelos. Essa reflexão também não pode descartar (como fazem os LARC e o pleito da FEBRASGO) a responsabilidade que os homens precisam ter em relação à contracepção e à proteção de doenças sexualmente transmissíveis (DST). Principalmente, não pode prescindir de considerar como um direito o fato de mulheres adolescentes iniciarem sua vida sexual antes do casamento. Em geral, essa iniciação amorosa-sexual torna-se um percurso mais complexo e cheio de obstáculos, muitos deles interpostos pelos adultos que não reconhecem tal experiência como necessária ao aprendizado como pessoa capaz de se autodeterminar ${ }^{24}$. Tampouco podemos ignorar o fato de que algumas destas adolescentes e jovens estejam em relações estáveis e tenham a maternidade como projeto de vida, num contexto social de extrema dificuldade de realização de outros projetos para seu futuro.

$\mathrm{O}$ aspecto mais doloroso da crítica à negligência e à indisciplina das mulheres no uso correto dos métodos contraceptivos é o fato de que essa falha, essa lacuna é transmutada para seu comportamento social, é identificada e julgada não como uma falha técnica, mas sim como uma falha individual de natureza moral. Sua incapacidade de seguir corretamente as prescrições médicas no uso de métodos contraceptivos - como ocorre no uso de outros medicamentos em tantos segmentos da população - compromete sua qualificação moral para decidir o que melhor the convém, sem necessariamente coincidir com a opinião dos especialistas.

A discussão sobre planejamento reprodutivo é sempre necessária mas nunca deve ocorrer isolada das relações de gênero que o determinam e muito menos tomando a mulher por sua condição de menoridade social. 


\section{Referências}

1. Brandao ER, Cabral CS. Da gravidez imprevista à contracepção: aportes para um debate. Perspectivas. Cad Saude Publica 2017; 33(2):e00211216.

2. Diniz D, Medeiros M, Madeiro A. Pesquisa Nacional de Aborto 2016. Cien Saude Colet 2017; 22(2):653-660.

3. Baum P, Fiastro A, Kunselman S, Veja C, Ricardo C, Galli B, Nascimento M. Garantindo uma resposta do setor de saúde com foco nos direitos das mulheres afetadas pelo vírus Zika. Cad Saude Publica 2016; 32:Epub03-Jun.

4. Galli B, Deslandes S. Ameaças de retrocesso nas políticas de saúde sexual e reprodutiva no Brasil em tempos de epidemia de Zika. Cad Saude Publica 2016; 32:EpubApr19.

5. Perpétuo IHO, Wong LLR. Desigualdade socioeconômica na utilização de métodos anticoncepcionais no Brasil: uma análise comparativa com base nas PNDS 1996 e 2006. In: Brasil. Ministério da Saúde (MS). Pesquisa Nacional de Demografia e Saúde da Criança e da Mulher-PNDS 2006: dimensões do processo reprodutivo e da saúde da criança. Brasília: MS; 2009. p. 87-104.

6. Brasil. Ministério da Saúde (MS). Pesquisa Nacional de Demografia e Saúde da Criança e da Mulher. PNDS 2006: relatório final. Brasília: MS, CEBRAP; 2008.

7. Comissão Nacional de Incorporação de Tecnologias no SUS (Conitec). Consultas públicas no 35 e no 36. [acessado 2017 Mar 3]. Disponível em: http://conitec.gov.br/ index.php/consultas-publicas-2015-encerradas

8. Penna IAA, Brito MB. A importância da contracepção de longo prazo reversível. Femina 2015; 43(1):1-6.

9. Sorpreso IC, Soares-Júnior JM, Baracat EC. Mulheres com vulnerabilidade sexual: anticoncepção reversível de longa duração pode ser a solução? Rev Bras Ginecol Obstet 2015; 37(9):395-396.

10. Higgins JA, Kramer RD, Ryder KM. Provider Bias in Long-Acting Reversible Contraception (LARC) Promotion and Removal: Perceptions of Young Adult Women. Am J Public Health 2016; 106(11):1392-1397.

11. Hillard PJA. What Is LARC? And Why Does It Matter for Adolescents and Young Adults? J Adolesc Health 2013; 52(Supl. 4):S1-5.

12. Berquó E, Lima LP. Planejamento da fecundidade: gravidezes não-desejadas - PNDS 1996 e 2006. In: Brasil. Ministério da Saúde (MS). Pesquisa Nacional de Demografia e Saúde da Criança e da Mulher - PNDS 2006: dimensões do processo reprodutivo e da saúde da criança. Brasília: MS; 2009. p. 135-149.

13. Federação Brasileira das Associações de Ginecologia e Obstetrícia (Febrasgo). Posicionamento da Comissão Nacional de Anticoncepção da Febrasgo sobre a decisão do MS [acessado 2017 Mar 3]. Disponível em: http://www.febrasgo.org.br/site/?p=12305.

14. Federação Brasileira das Associações de Ginecologia e Obstetrícia (Febrasgo). Portarias $n^{\circ} 12$ e $n^{\circ} 13$, de 11 de abril de 2016, da Secretaria de Ciência, Tecnologia e Insumos Estratégicos do Ministério da Saúde. [acessado 2017 Mar 3]. Disponível em: http://www.febrasgo.org. $\mathrm{br} / \mathrm{site} / \mathrm{p}=12305$
15. Lubianca JN. Opções de Anticoncepção na Adolescência. In: Carvalho FD, Wannmacher L, organizadores. Uso racional de medicamentos: fundamentação em condutas terapêuticas e nos macroprocessos da Assistência Farmacêutica. Brasília: Organização Pan-Americana da Saúde (OPAS), Organização Mundial da Saúde (OMS) no Brasil; 2016. 1(17)

16. Moreau C, Cleland K, Trussell J. Contraceptive discontinuation attributed to method: dissatisfaction in the United States. Contraception 2007; 76(4):267-272.

17. Moreau C, Bouyer J, Bajos N, Rodríguez G, Trussell J. Frequency of discontinuation of contraceptive use: results from a French population-based cohort. Human Reproduction 2009; 1:1-6.

18. Brandão ER, Cabral CS, Ventura M, Paiva SP, Bastos LL, Oliveira NVBV, Szabo I. "Bomba hormonal": os riscos da contracepção de emergência na perspectiva dos balconistas de farmácias no Rio de Janeiro, Brasil. Cad Saude Publica 2016; 32(9):e00136615.

19. Brandão ER, Cabral CS, Ventura M, Paiva SP, Bastos LL, Oliveira NVBV, Szabo I. Os perigos subsumidos na contracepção de emergência: moralidades e saberes em jogo. Horizontes Antropológicos 2017; 23(47):131-161.

20. Cleland K, Raymond EG, Westley E, Trussell J. Emergency contraception review: evidence-based recommendations for clinicians. Clin Obstet Gynecol 2014; 57(4):741-750.

21. Westley E, Glasier A. Emergency contraception: dispelling the myths and misperceptions. Bull World Health Organ 2010; 88:243.

22. Figueiredo R, Borges ALV, Paula SHB, organizadoras. Panorama da contracepção de emergência no Brasil. São Paulo: Instituto de Saúde; 2015.

23. Lago TG, Kalckmann AS, Alves MCGP, Barbosa RM, Escuder ML, Olosen J. Práticas contraceptivas na cidade de São Paulo: prevalência, necessidades não atendidas e atuação do SUS. "Ouvindo mulheres: contracepção no município de São Paulo" [relatório científico final]. São Paulo: Instituto de Saúde, Secretaria de Estado de Saúde de São Paulo; 2016.

24. Brandão ER. Iniciação sexual e afetiva: exercício da autonomia juvenil. In: Heilborn ML, organizadora. Família e sexualidade. Rio de Janeiro: FGV; 2004. p. 63-86.

Artigo apresentado em 11/03/2017

Aprovado em 22/05/2017

Versão final apresentado em 24/05/2017 
